\title{
Images
}

\section{White cerebellum sign: red flag in the emergency department}

\author{
Divya Singh, MBBS, MD*; Amit Sharma, MBBS, MD†
}

A 3-year-old boy presented to the emergency department with fever, generalized tonic-clonic seizures, and altered sensorium within 6 hours of administration of the diphtheria, pertussis, and tetanus (DPT) vaccine. The child had features suggestive of raised intracranial pressure with papilledema on fundoscopic examination. Contrast-enhanced computed tomography (CT) of the head (Figure 1) revealed diffuse hypodense attenuation of bilateral cerebral hemispheres with effacement of the basal cisterns and sulcal spaces. The cerebellum showed normal attenuation, thus appearing hyperdense relative to the cerebral parenchyma. This is known as the white cerebellum sign. Despite the best possible efforts, the patient passed away.

The white cerebellum sign is a pointer toward severe hypoxic ischemic brain injury. ${ }^{1}$ It has been described in conditions such as birth asphyxia, head injury, drowning, status epilepticus, hypothermia, meningoencephalitis, and other causes of global hypoperfusion. The white cerebellum sign results from diffuse cerebral edema leading to loss of normal grey-white matter differentiation of the cerebral parenchyma. It is postulated that there is preferential blood flow to the posterior fossa structures in severe anoxia, thereby preserving the normal appearance of the cerebellum, making it look brighter on CT evaluation. The white cerebellum sign suggests a poor prognosis indicative of irreversible brain damage. ${ }^{2}$ Knowledge of this sign is important from a diagnostic, therapeutic, and prognostic standpoint.

\section{Competing interests: None declared.}

Keywords: contrast-enhanced computed tomography; diphtheria, pertussis, and tetanus (DPT) vaccine; white cerebellum sign

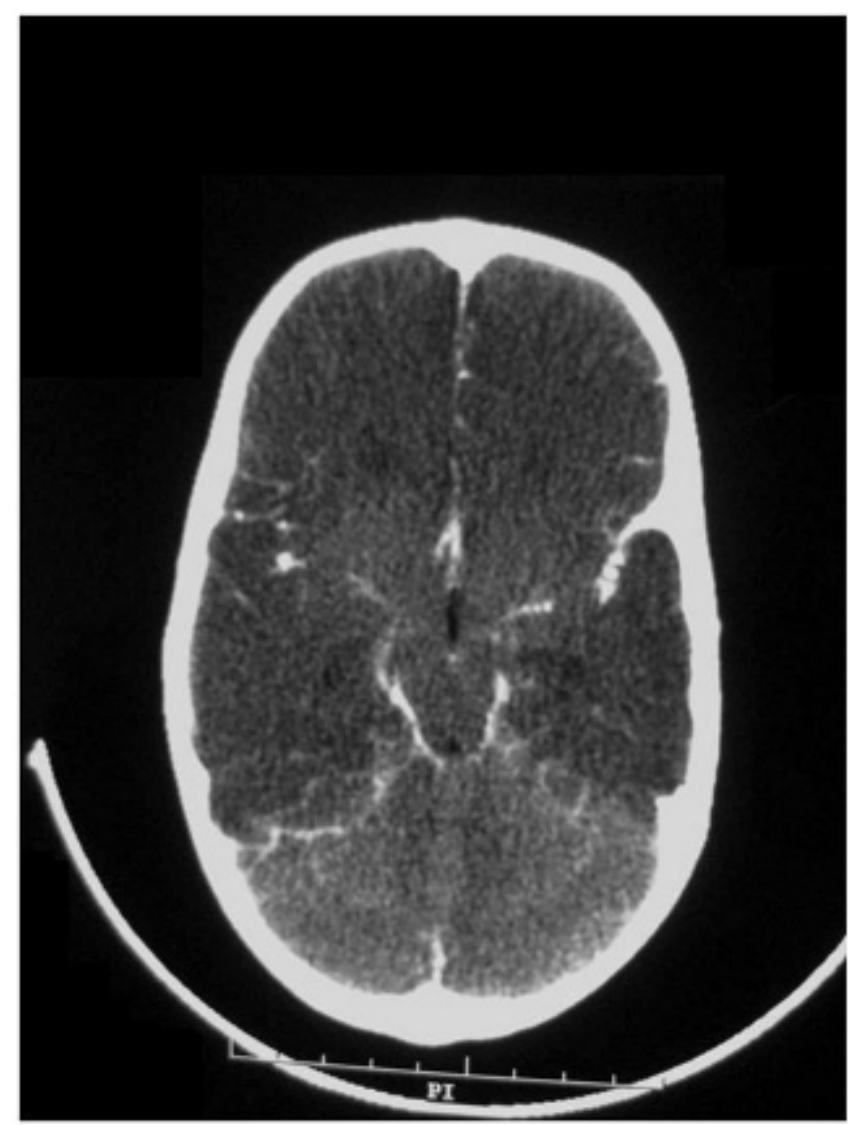

Figure 1. Computed tomographic scan showing hyperdense cerebellum relative to the cerebral hemispheres.

\section{REFERENCES}

1. Huang BY, Castillo M. Hypoxic-ischemic brain injury: imaging findings from birth to adulthood. Radiographics 2008; 28:417-39, doi:10.1148/rg.282075066.

2. Han BK, Towbin RB, De Courten-Myers G, et al. Reversal sign on CT: effect of anoxic/ischemic cerebral injury in children. AfNR Am 7 Neuroradiol 1989;10:1191-8.

From the *Department of Radiodiagnosis, All India Institute of Medical Sciences, New Delhi, India; †Department of Nephrology, Fortis Hospital, Mohali, Punjab, India.

Correspondence to: Dr. Divya Singh, Department of Radiodiagnosis, All India Institute of Medical Sciences, New Delhi, India 110029; docdivyas@ yahoo.co.in.

This article has been peer reviewed.

(C) Canadian Association of Emergency Physicians 